\title{
POSITIVE EFFEKTE SOZIALEN FAULENZENS BEIM LÖSEN KOMPLEXER PROBLEME*
}

Annette Feuchter und Joachim Funke

Zusammenfassung: Mit dem Begriff „Soziales Faulenzen“ sind Motivations- und Leistungsverluste in Gruppen bezeichnet worden, die durch sinkende Verantwortlichkeit der Gruppenteilnehmer für das Leistungsergebnis entstehen. Neuere Studien ließen daran Zweifel aufkommen und postulierten ein paradoxes Verhältnis von Motivation und Gruppenleistung derart, dass bei geringerer Motivation höhere Leistung zu erwarten sei. Die vorliegende Arbeit dient der Klärung dieser Frage. Es werden zwei Experimente berichtet, in denen jeweils 60 Personen in Dreiergruppen ein computersimuliertes Waldbrand-Szenario (Networked Fire Chief) bearbeiteten. Variiert wurden die Schwierigkeit (leichte versus schwierigere Version) sowie die Verantwortlichkeit (koaktiv versus kollektiv). Während in Experiment 1 die Teilnehmenden nur auf dem ihnen zugewiesenen Spielfeld-Teil agieren konnten, konnten sie in Experiment 2 auf allen Teilfeldern agieren. Gemessen wurden Anstrengung und Leistung auf individueller wie Gruppenebene. Im Ergebnis zeigt sich unter Bedingungen kollektiver Verantwortlichkeit erwartungsgemäß ein Nachlassen der Anstrengung. Interessanterweise führt dies jedoch nicht zu einem Abfall der Leistung; unter der schwierigeren Bedingung zeigt sich vielmehr paradoxerweise eine erhöhte Leistung bei sinkender Anstrengung. Diskutiert werden die Konsequenzen für die Theorie kollektiver Anstrengung von Karau und Williams, deren Modell um weitere Einflussfaktoren ergänzt werden muss.

\section{Einleitung}

Die meisten Aufgaben des täglichen Lebens können nur in und von Gruppen bewältigt werden. Eine Vielzahl dieser Gruppenaufgaben sind kollektive Aufgaben, in der die individuellen Beiträge zu einem Gesamtergebnis zusammengefasst werden. In der Regierung, in zahlreichen Komitees und Kommissionen, in der Wirtschaft sowie im Sport erleben wir stets, dass kollektive Entscheidungen getroffen und kollektive Leistungen erbracht werden, die ein Produkt individueller Beiträge darstellen.

Wie lassen sich solche komplexen Aufgaben effizient durch die Gestaltung der Arbeitsstruktur lösen? Wirkt die Arbeit in Gruppen motivierend oder nicht? Wie verhält sich die Motivation zu den erbrachten Leistungen?

\footnotetext{
* Die hier berichtete Arbeit entstammt der Dissertation der Erstautorin, die vom Zweitautor betreut und begleitet wurde. Eine ausführliche Darstellung der Studie findet sich bei Feuchter (2001). Die Durchführung der Experimente wurde durch ein Stipendium der Jutta-Heidemann-Stiftung ermöglicht, wofür auch an dieser Stelle nochmals gedankt wird. Für wertvolle Kommentare und Anregungen zu einer früheren Fassung des Manuskripts danken wir den Herausgebern der KZfSS sowie zwei anonymen Gutachtern.
} 
In der Literatur zur Frage, wie sich das Verhältnis von Motivation und Leistung in Gruppen erklären lässt, finden sich mindestens die folgenden vier Standpunkte.

1. Motivationssteigerung durch Übersummativität. Mit den Klassikern der Soziologie wie Emile Durkheim und Georg Simmel lässt sich ein positiver Einfluss der Gruppe dadurch begründen, dass diese mehr als die Summe ihrer Teile ist. Hier wird ein Synergieeffekt vermutet, der sich positiv auf die Güte der Leistungen auswirkt. Auch im Zuge der aktuellen Renaissance von Gruppenarbeit in Arbeitskontexten wird nachdrücklich auf den Synergieeffekt der Gruppen hingewiesen und dessen motivationssteigernde Wirkung betont (Hackman und Morris 1975; Hertel 2000; Hertel, Kerr und Messé 2000; Nahavandi und Aranda 1994; Warnecke 1997). Wie Reimer (2001; vgl. auch Reimer et al. 1997) ausführt, gibt es unter bestimmten Bedingungen Leistungsvorteile, die man durch gesteigerte Motivation und die Möglichkeit, die Fehler einzelner in der Gruppe kompensieren zu können, erklären kann.

2. Motivationsverlust mit negativen Auswirkungen: Soziales Faulenzen. Neben diesen positiven Befunden liegen aber auch Ergebnisse vor, die den postulierten Synergieeffekt in Gruppen bezweifeln (Brown 2000; Hill 1982). Empirisch wurde etwa nachgewiesen, dass die Leistungen von Personen in Gruppen häufig geringer ausfallen als von Personen, die allein für die Erstellung eines Produktes verantwortlich sind (Arnscheid, Diehl und Stroebe 1997; Harkins und Szymanski 1988, 1989; Shepperd 1993; Shepperd und Taylor 1999). Personen, so die Begründung, schöpften ihr Leistungspotenzial in Gruppen nicht aus, da die individuellen Beiträge nicht zurechenbar seien. Dieser Motivationsverlust wird auch als soziales Faulenzen (social loafing) bezeichnet (Karau und Williams 1993, 1995, 1997, 2000; Latané, Williams, und Harkins 1979).

3. Unabhängigkeitshypothese. Weiterhin liegen Untersuchungen vor, die die universelle Gültigkeit des Auftretens von sozialem Faulenzen in Zweifel ziehen (Brickner et al. 1986; Harkins und Petty 1982). Diesen Befunden zufolge wird die Leistung im Falle subjektiv bedeutsamer Aufgaben nicht dadurch beeinflusst, ob diese in Gruppen oder alleine erbracht werden. Sind Akteure intrinsisch motiviert, führen unterschiedliche situative Anreize den Annahmen dieser Position folgend weder zu einer Steigerung noch zu einer Minderung der Leistung (Shepperd 1993; Szymanski und Harkins 1993). Ihre Vertreter lehnen demnach sowohl die These, auf Grund des Synergieeffektes in Gruppen komme es zu einer Leistungssteigerung, als auch jene ab, es komme zu einem Motivationsverlust und damit zu Leistungseinbußen.

4. Motivationsverlust mit positiven Auswirkungen. Im Unterschied zu diesen drei Positionen gibt es mittlerweile auch Evidenzen für ein Auseinandertreten zwischen Motivation und Leistung in Gruppen. Einzelne Studien haben nachgewiesen, dass im Falle subjektiv bedeutsamer Aufgaben ein paradoxes Verhältnis von Motivation und Leistung vorliegt. Demnach werden in Gruppen - selbst wenn Personen weniger motiviert sind - bessere Leistungen erzielt als unter individuellen Arbeitsbedingungen (Bartis et al. 1988; Griffith et al. 1989; Jackson und Williams 1985). Gruppen, in denen Motivationsverluste auftreten, können somit sogar zu besseren Leistungen gelangen als hoch motivierte Personen. 
Dieses Auseinandertreten von Anstrengung und Leistung lässt sich auf die in der Yerkes-Dodson-Regel beschriebene Gesetzmäßigkeit zurückführen, der zufolge unter individuellen Arbeitsbedingungen ein erhöhtes Erregungsniveau festzustellen ist (Yerkes und Dodson 1908). Nach dem Yerkes-Dodson-Gesetz besteht zwischen dem allgemeinen Erregungsniveau, das sich unmittelbar auf die Anstrengung auswirkt, und der Leistung eine umgekehrte U-förmige Beziehung. Die Leistung ist demnach bei einem mittleren Erregungsniveau optimal, wohingegen eine zu niedrige und eine zu hohe Erregung zu einer suboptimalen Leistung führen. Die Validität dieses Gesetzes lässt sich vor allem beim Bearbeiten schwieriger Aufgabenstellungen nachweisen, zumal eine erhöhte Anstrengung bei nicht routinisiertem Verhalten ein Ansteigen von Fehlern wahrscheinlich werden lässt (Jackson und Williams 1985).

Da die Forschung zum sozialen Faulenzen bislang zur Validierung des so genannten Ringelmann-Effektes ${ }^{1}$ meist einfache Aufgabenstellungen, wie z.B. das gemeinsame Ziehen an einem Seil, verwendet hat, war eine Trennung zwischen Anstrengung und Leistung nicht erforderlich. Die Anstrengung galt sowohl als Indikator für die Motivation als auch für die Leistung. Im Falle schwieriger bzw. komplexer Aufgaben kommt es hingegen zu einem Auseinandertreten beider Größen, was eine Unterscheidung von Motivation und Leistung erforderlich werden lässt.

Stellt man sich vor dem Hintergrund dieses Problemaufrisses die Frage nach einer effizienten Organisation von Arbeitsabläufen, zeigt sich, wie schwierig es ist, diese zu beantworten. Die vier sich ausschließenden Positionen lassen deutlich werden, dass nach wie vor unklar ist, wie Arbeitszusammenhänge effizient zu gestalten sind, um eine optimale Bewältigung komplexer Aufgaben sicherstellen zu können. Vor diesem Hintergrund ergibt sich die Notwendigkeit einer Untersuchung, die Aufschluss darüber gewährt, wie sich unterschiedliche Arbeitsbedingungen und Aufgabenstellungen auf die Motivationsformen der Akteure einerseits und auf die Güte der kollektiven Leistung andererseits auswirken. Solange ungeklärt ist, ob motivationsfördernde Maßnahmen erstens das individuelle Leistungspotenzial erhöhen und ob diese zweitens eine effizientere Bewältigung der Aufgaben gewährleisten, lässt sich die Implementierung entsprechender Maßnahmen immer nur bedingt rechtfertigen.

Die vorliegende experimentelle Studie versucht diese Forschungslücke zu schließen, indem sie die Auswirkungen unterschiedlicher Arbeitsbedingungen auf die Motivation und Leistung beim Lösen eines komplexen Problems untersucht. Es wird davon ausgegangen, dass koaktive und kollektive Arbeitsbedingungen in stärkerem oder in schwächerem Maße das Bedürfnis bei den Personen erzeugen, sich anzustrengen, um be-

1 Nach Ringelmann wenden Personen, sofern sie gemeinsam an einem Seil ziehen, weniger Kraft auf, als wenn sie alleine an dem Seil zögen. Zur Validierung seiner These verglich Ringelmann die Leistungen von Einzelpersonen mit den Leistungen von Gruppen unterschiedlicher Personenzahlen. Wie die Untersuchung nachweisen konnte, nahm der Kraftaufwand einzelner Personen beim Hinzukommen jeder weiteren Person proportional zur Anzahl der Personen ab. Auf Grund des Zusammenhangs zwischen Kraftaufwand und Gruppengröße wurde zunächst die Anzahl der Personen als Mediator für die Erklärung des Prozessverlustes bestimmt. War Ringelmann der Überzeugung, der geringere Kraftaufwand sei reinen Koordinationsverlusten geschuldet, so wird in Anlehnung an Steiner (1972) davon ausgegangen, dass neben den Koordinationsverlusten in dieser Untersuchung auch Motivationsverluste auftraten, die ebenfalls einen Einfluss auf die Produktivität hatten (Kravitz und Martin 1986). 
stimmte Ziele zu erreichen. Koaktiv heißt eine Arbeitsbedingung, bei der zwar nominell eine Gruppe die gegebene Aufgabe bearbeitet, aber die Einzelleistungen dennoch identifizierbar bleiben (so genannte Nominalgruppe). Kollektiv dagegen nennt man Gruppenarbeit, bei der die Einzelanteile nicht mehr identifiziert werden (so genannte Realgruppe).

Die Aufgabe der Teilnehmenden besteht darin, in Dreiergruppen unter Vorgabe der jeweiligen Arbeitsbedingung ein computersimuliertes Szenario zu bearbeiten. Der Einsatz dieses Szenarios ermöglicht es, die Komplexität der Problemstellung - und damit auch deren subjektive Bedeutsamkeit (vgl. Brickner et al. 1986) - kontrolliert zu manipulieren, um den Einfluss verschieden komplexer Probleme auf Motivation und Leistung zu untersuchen. Dadurch lässt sich feststellen, ob beim Lösen komplexer Probleme soziales Faulenzen überhaupt auftritt und wenn ja, wie sich dieser Motivationsverlust bei gesteigerter Komplexität des Problems auf die Güte der kollektiven Leistung auswirkt.

Doch bevor Untersuchungsmethode und Ergebnisse dargestellt und diskutiert werden, sollen kurze Ausführungen über ein einschlägiges Modell kollektiven Aufwands sowie über daraus resultierende offene Fragen erfolgen.

\section{Collective Effort Model (CEM)}

In der Motivationsforschung dominieren wert-erwartungstheoretische Modelle die Theoriebildung. Ein solches Modell, das_Collective Effort Model (CEM), welches auf der Grundlage einer Metaanalyse in der Forschung zum sozialen Faulenzen (Karau und Williams 1993) entwickelt wurde, bildet die theoretische Grundlage der vorliegenden Untersuchung. Das CEM stellt einen Versuch dar, alle bislang in der Forschung ausgemachten validen Bedingungen für soziales Faulenzen in einem einzigen Modell zu integrieren.

Dem Modell liegt die These zu Grunde, dass sich Personen im Falle von kollektiven Leistungssituationen auf Grund der geringeren wahrgenommenen Instrumentalität weniger anstrengen als in individuellen oder koaktiven Leistungssituationen. Karau und Williams (1993) begründen ihre These dadurch, dass die wahrgenommene Instrumentalität (d.h. die Beziehung zwischen Handlungsfolgen und Handlungsergebnisfolgen) in kollektiven Leistungssituationen im Vergleich zu individuellen oder koaktiven Leistungssituationen komplexer und ungewisser sei.

Die Autoren orientieren sich in ihrer Analyse an Vrooms (1964) Instrumentalitätstheorie, die er im Rahmen seiner Untersuchung zur Arbeitszufriedenheit entwickelte. In der Tradition der Wert-Erwartungstheorie stehend hat Vroom eine Instrumentalitätstheorie vorgelegt, die arbeitspsychologische Befunde zur Arbeitszufriedenheit und Arbeitsleistung integriert. Dieser Theorie folgend wird das Handeln durch die Antizipation bestimmter Konsequenzen beeinflusst, die auch als Outcomes oder als Handlungsergebnisfolgen bezeichnet werden. Die Folgen des Handelns stellen für die Handelnden positive oder auch negative Anreizwerte dar. Sie werden von Personen antizipiert und motivieren dementsprechend auch das Handeln. Die Motivation ist Vroom folgend abhängig von: 
a) der Erwartung (expectancy), die besagt, eine hohe Anstrengung führe zu hohen Leistungen;

b) der Instrumentalität (instrumentality), die besagt, dass gute Leistungen auch entsprechend honoriert werden und durch das Erbringen guter Leistungen individuelle Ziele realisiert werden können, und

c) der Bewertung der Resultate bzw. Ergebnisse (valence of outcomes), die besagt, die Ergebnisse würden als wünschenswert betrachtet werden (Vroom 1964: 19f.).

Für kollektive Leistungssituationen ergänzen Karau und Williams (1993) dieses Modell durch eine Spezifizierung der Instrumentalität. Die Instrumentalität wird in kollektiven Situationen determiniert: a) durch die wahrgenommene Beziehung zwischen individueller und kollektiver Leistung, b) durch die wahrgenommene Beziehung zwischen Gruppenleistung und Gruppenoutcome sowie c) durch die wahrgenommene Beziehung zwischen Gruppenoutcome und individuellem Outcome. ${ }^{2}$

Demnach ist die wahrgenommene Instrumentalität Karau und Williams (1993) zufolge in kollektiven Leistungssituationen niedriger als in koaktiven Leistungssituationen. In diesem Zusammenhang vertreten die Autoren zwei Thesen: Der ersten These (sinkende Instrumentalität) folgend besteht in der individuellen Leistungssituation ein stärkerer Zusammenhang zwischen der wahrgenommenen Anstrengung und dem individuellen Ergebnis. In der kollektiven Situation ist dieser Zusammenhang hingegen nicht unmittelbar gegeben. Soziales Faulenzen ist darauf zurückzuführen, dass andere Faktoren als die individuelle Anstrengung die Leistung beeinflussen, weshalb die wahrgenommene Instrumentalität der Mittel sinkt. Nach der zweiten These (gleichmäßige Ergebniszuschreibung) ist in einer individuellen Leistungssituation das Ergebnis unmittelbar mit der eigenen Leistung verknüpft. In einer kollektiven Situation wird das Gruppenprodukt unter den einzelnen Gruppenmitgliedern aufgeteilt. Besteht in der individuellen Situation eine analytische, d.h. eine individuell durchschaubare Verknüpfung zwischen der Anstrengung und den individuellen Konsequenzen, so liegt in der kollektiven Situation eine synthetische, d.h. eine nicht individuell durchschaubare Verknüpfung vor. Soziales Faulenzen verdankt sich dem Umstand, dass andere Faktoren als die individuelle Leistung das individuelle Ergebnis beeinflussen und das erzeugte Gruppenprodukt unter den Gruppenmitgliedern aufgeteilt wird.

Es liegt nahe, die Überlegungen von Karau und Williams im Sinne der Theorie kollektiven Handelns zu deuten (vgl. Olson 1992; Ostrom 1997). Motivationsverluste lassen sich dieser zufolge durch das Trittbrettfahrerproblem erklären, das sich bei der Erstellung kollektiver Güter ergibt. Ist der einzelne Beitrag einer Person zur Lösung eines Problems nicht identifizierbar, so stellt jeder individuelle Beitrag ein Kollektivgut dar. Da es jeder Person möglich ist, den erbrachten Beitrag sich selbst zuzurechnen, gilt für jeden Beitrag, dass er die Eigenschaften der Nichtrivalität im Konsum und der Nichtausschließbarkeit aufweist. Motivationsverluste lassen sich demnach als Trittbrettfahren interpretieren, denn es ist für jede beteiligte Person rational, die entsprechenden Beiträge nicht selbst zu erbringen, sondern sich die Beiträge der anderen als eigene Leistungen zuzurechnen.

2 Vor dem Hintergrund des unter Abschnitt II dargestellten Versuchsaufbaus ist davon auszugehen, dass die Versuchspersonen das Löschen der Feuer als eine angestrebte Handlungsfolge betrachten. 
Zahlreiche Studien haben die theoretischen Annahmen des CEM auf deren empirische Gültigkeit überprüft und die Richtigkeit für das Bearbeiten einfacher Aufgabenstellungen bestätigt (Arnscheid et al. 1997; Hoeksema-van Orden, Gaillard und Buunk 1998; Karau und Williams 1993, 1995; Shepperd und Taylor 1999). Die Prognosefähigkeit des CEM für das Lösen schwieriger und komplexer Aufgabenstellungen ist hingegen noch ungeklärt. Daher stellt sich die Frage, ob das Modell auch Aussagen über das Auftreten von sozialem Faulenzen beim Lösen komplexer Probleme erlaubt.

\section{Anwendung des CEM auf komplexe Probleme: Erste Hypothesen}

Einerseits ist für komplexe Probleme auf Grund der Affinität zu subjektiv bedeutsamen Aufgabenstellungen (vgl. Brickner et al. 1986) zu erwarten, dass die Bewertung der Konsequenzen hoch ist und Personen ihren Beiträgen bei der Bearbeitung dieser Probleme einen besonderen Wert beimessen. Demnach würden in diesem Falle geringere Motivationsverluste auftreten. Andererseits ist dem CEM folgend zu erwarten, dass die wahrgenommene Instrumentalität in der kollektiven Situation geringer als in der koaktiven Situation ist. Demnach tritt auch beim Lösen komplexer Probleme soziales Faulenzen auf.

Das wert-erwartungstheoretische CEM ist eine Motivationstheorie. Dem Modell liegt die Annahme zu Grunde, dass die Motivation die Leistung positiv beeinflusst und eine erhöhte Motivation zu einer gesteigerten Leistung führt. Demzufolge kann das CEM ein inverses Verhältnis von Motivation und Leistung auch nicht erklären.

Wie bei Karau und Williams (1993) findet sich auch in jenen Studien, die keine Motivationsverluste unter kollektiven Arbeitsbedingungen ausmachen konnten, keine Unterscheidung zwischen Anstrengung und Leistung. Die Frage, weshalb Brickner et al. (1986) sowie Harkins und Petty (1982) zu dem Befund gelangten, soziales Faulenzen trete beim Lösen subjektiv bedeutsamer Aufgaben nicht auf, lässt sich dadurch beantworten, dass gerade nicht die Anstrengung als Maßstab für soziales Faulenzen zu Grunde gelegt wurde, sondern von der Leistung auf die Motivation rückgeschlossen wurde. Auf Grund der identischen Leistungen unter kollektiven und koaktiven Situationen gelangten die Studien zu dem Ergebnis, es gebe im Falle dieser Aufgaben keine Motivationsverluste. Tatsächlich könnten aber Anstrengungsunterschiede vorgelegen haben.

Für die weitere Analyse wird deshalb die Trennung zwischen Motivation und Leistung grundlegend sein. Dies erlaubt es auch, soziales Faulenzen ausschließlich mit Motivationsverlusten und nicht mit Leistungseinbußen zu identifizieren. Unter Berücksichtigung dieser Trennung soll also der Frage nachgegangen werden, inwiefern der wert-erwartungstheoretische Erklärungsrahmen des CEM, dessen Gültigkeit mittlerweile bei einfachen Aufgaben mehrfach bestätigt wurde, auch bei komplexeren kollektiven Anforderungen Bestand hat oder ob dieses im Falle der Prognosefähigkeit für die Leistung beim Lösen komplexer Probleme durch nicht-kognitive Faktoren zu ergänzen sein wird.

Wie das Kausalmodell in Abbildung 1 zeigt, lässt sich das inverse Verhältnis zwischen Motivation und Leistung dadurch erklären, dass die Leistung neben bewussten 
Abbildung 1: Kausalmodell: Einflussfaktoren auf die Motivation und Leistung

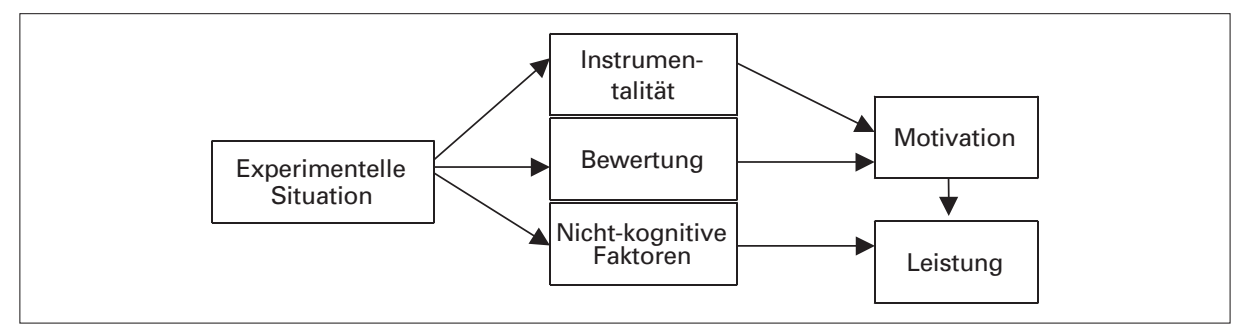

Entscheidungen für die Ausführung von Handlungen auch durch nicht-kognitive Faktoren (z.B. Stress, irrelevante Informationsverarbeitung) beeinflusst wird. Damit entstehen situationsbedingt nicht-intendierte Handlungsfolgen, die im Falle der Bearbeitung eines komplexen Problems die kollektive Leistung herabsetzen können.

\section{Methode}

In einer experimentellen Studie sollen die theoretischen Annahmen über das Auftreten von sozialem Faulenzen und die Auswirkungen auf die Leistung beim Lösen komplexer Probleme auf ihre Gültigkeit geprüft werden. Hierfür wurde das computersimulierte Szenario Networked Fire Chief (Omodei und Wearing 1993, 1995a, 1995b) zu Grunde gelegt, bei dem die Teilnehmenden in Dreiergruppen unter Vorgabe unterschiedlicher Versuchsbedingungen einen simulierten Waldbrand zu bekämpfen haben (zur Verwendung computersimulierter Szenarien in der Problemlöseforschung siehe Funke 2003). In diesem Abschnitt wird zunächst das Szenario genauer beschrieben; außerdem werden die unabhängigen und abhängigen Variablen sowie die Hypothesen dargestellt, Angaben zu den Versuchspersonen gemacht und die Versuchsdurchführung dargelegt.

\section{Das Szenario Networked Fire Chief}

Bei dem von der australischen Forschergruppe um Mary Omodei und Alexander Wearing (1993, 1995a, 1995b) entwickelten computersimulierten Szenario Networked Fire Chief handelt es sich um die Simulation einer realen Feuerlöschsituation. Die Aufgabenstellung für die Probanden besteht darin, dass jeweils drei Personen gemeinsam mit den ihnen zur Verfügung stehenden Löschgeräten (Feuerwehrautos und Hubschrauber) einen Flächenbrand bekämpfen müssen, der in bestimmten Segmenten auf der Bildschirmoberfläche ausbricht. In Anlehnung an Steiners Taxonomie handelt es sich beim gemeinsamen Bekämpfen der Feuer um eine additive Optimierungsaufgabe (Steiner 1972). Ist ein Feuer ausgebrochen, so breitet es sich mit einer zuvor festgelegten Geschwindigkeit auf die angrenzende Fläche aus. Die Ausbreitungsrichtung wird durch die Windrichtung bestimmt, welche sich - unter Kontrolle der Versuchsleiterin - im Laufe des Spiels häufiger verändert. Abbildung 2 zeigt den Bildschirmaufbau für die Versuchspersonen. 
Abbildung 2: Display der Feuer-Situation für eine der drei Versuchspersonen: Im fortgeschrittenen Stadium sind bereits Teile der Fläche verbrannt (schwarz mit rotem Feuerrand), blau ist der Wasservorrat zum Auftanken der Löschfahrzeuge; diese sind im oberen linken Teil von den Flammen eingeschlossen, im rechten Teil dagegen auf sicherem Grund. Die Aufteilung der Gesamtfläche in 3 Segmente ist gut zu erkennen

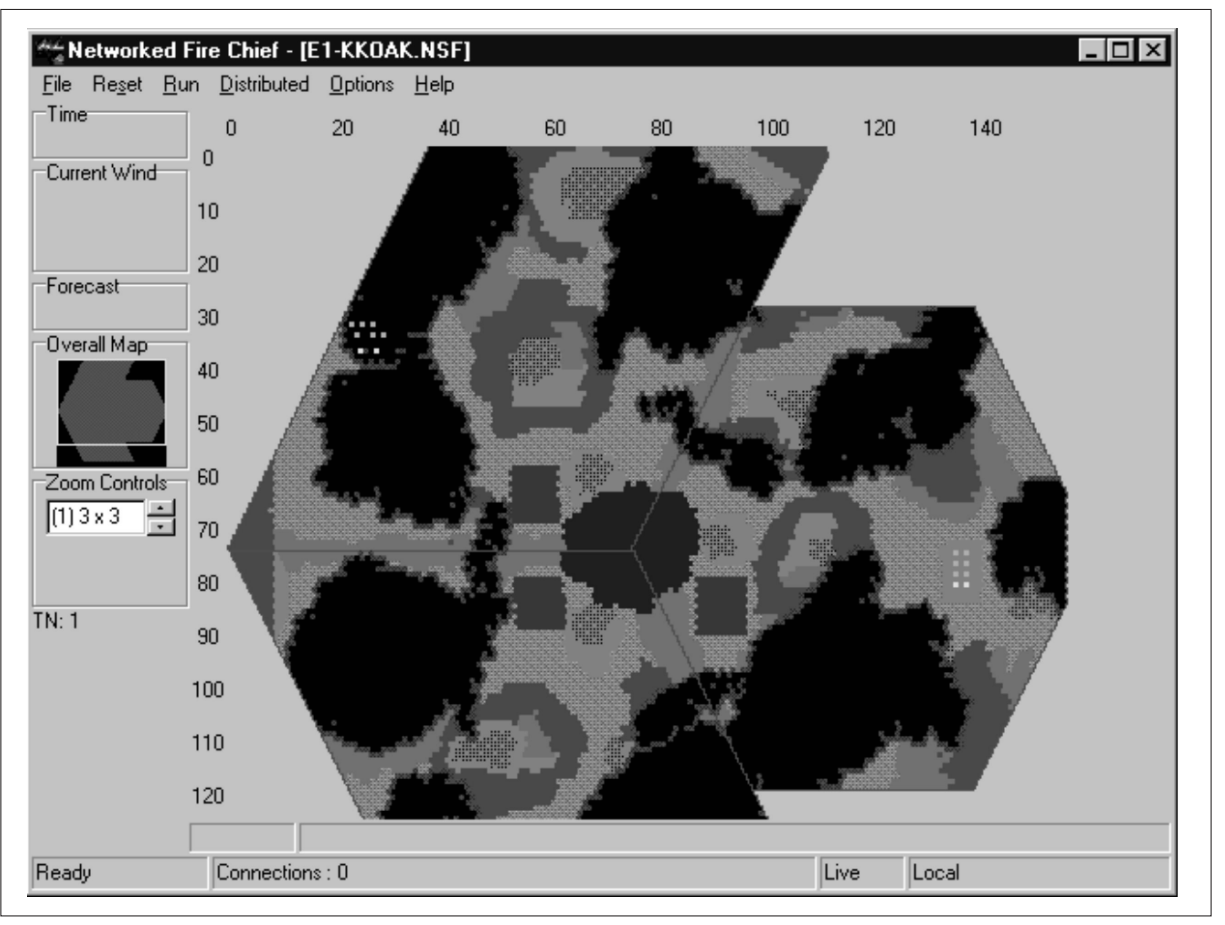

Auf dem Spielfeld befinden sich durch unterschiedliche Symbole markierte Objekte (besiedelte Gebiete, Vegetationen), die es zu retten gilt. Sie unterscheiden sich hinsichtlich ihrer Entflammbarkeit und weisen jeweils einen unterschiedlichen Stellenwert im Hinblick auf die Gewichtung zum Gesamtergebnis auf. So ist es beispielsweise wichtiger, besiedelte Gebiete als Grünflächen zu retten. Des Weiteren befinden sich auf der Bildschirmfläche zwei unterschiedliche Ressourcendepots. Die Anzahl der Ressourcen, die einerseits zur Feuerbekämpfung (Wasser) und andererseits zur Fortbewegung der Löschgeräte (Benzin) erforderlich sind, bestimmen das Komplexitätsniveau der Szenarien. Für die Untersuchung, die zwischen einem einfachen und einem komplexen Szenario unterscheidet, benötigen die Löschgeräte im einfachen Szenario lediglich Wasser zum Feuerlöschen, im komplexen Szenario benötigen die Geräte zusätzlich Benzin, um sich fortbewegen zu können. 


\section{Unabhängige und abhängige Variablen}

Generelles Ziel der Manipulation von Versuchsbedingungen war es, die Einflüsse von drei Faktoren, 1. der Arbeitsbedingung (kollektiv vs. koaktiv), 2. dem Komplexitätsniveau des Problems (einfach vs. komplex) und 3. der Interaktion (ohne vs. mit Interaktion) auf die Motivation und Leistung zu überprüfen. Im ersten Experiment konnten die Probanden nur auf ihrem Spielfeld die Feuer bekämpfen, wohingegen es ihnen im zweiten Experiment auch möglich war, auf den angrenzenden Feldern die Feuer zu bekämpfen. Dies impliziert, dass lediglich im zweiten Experiment interagiert wurde und in den Realgruppen Koordinationsverluste auftreten konnten, die eine Herabsetzung der Anstrengung beim Löschen der Feuer zur Folge haben könnten.

Die Arbeitsbedingung beinhaltet, dass den Probanden in der koaktiven Situation mitgeteilt wurde, die Versuchsleiterin sei daran interessiert, wie gut die einzelnen Spieler auf ihren jeweiligen Feldern die Feuer bekämpfen. In der kollektiven Leistungssituation wurde den Probanden mitgeteilt, dass es nicht möglich sei, die individuellen Leistungen zu beurteilen, sondern das Interesse daran bestünde, wie gut die gesamte Gruppe die Feuer insgesamt bekämpft. Zudem erhielten die Spieler unter beiden Arbeitsbedingungen kein explizites Feedback über ihre individuellen bzw. kollektiven Leistungen, um eine Wettbewerbssituation zu unterbinden.

Das Komplexitätsniveau des Problems wurde dahingehend manipuliert, dass die Probanden im Falle des einfachen Problems lediglich darauf achten mussten, ob ihre Löschgeräte über ausreichend Wasser verfügten. Im Falle des komplexen Problems bestand die Herausforderung hingegen darin, die zum Einsatz gebrachten Löschgeräte mit einer weiteren Ressource Benzin zu versorgen. Auf Grund der höheren Abhängigkeiten der Löschfahrzeuge von den Ressourcen steigen die Komplexität des Problems und damit auch die Anforderungen an die Problemlöser bei der Bekämpfung der Brände.

Die Interaktion wurde als Between-Subjects-Faktor in Form von zwei aufeinander folgenden Experimenten realisiert, die beiden anderen als Within-Subjects-Faktoren (Messwiederholung).

Interaktion. Für das erste Experiment war vorgesehen, dass die drei beteiligten Spielpartner nicht auf den benachbarten Flächen intervenieren können, sondern nur die eigene, ihnen zugewiesene Fläche bearbeiten konnten. Für das zweite Experiment hingegen ist auch eine Intervention auf den Flächen der benachbarten Spieler durchgängig erlaubt. Dadurch erhöht sich auch die Komplexität der sozialen Situation im zweiten gegenüber dem ersten Experiment.

Aus der Forschung zum komplexen Problemlösen in sozialen Situationen hat sich ergeben, dass eine Variation unterschiedlicher Interaktionsformen geboten ist, will man Aussagen über situative Einflüsse auf die Motivation und Leistung unter realitätsnäheren Bedingungen treffen. Für den Einsatz dieses Szenarios spricht, dass die Untersuchungsergebnisse Aussagen darüber erlauben, ob soziales Faulenzen auch beim Lösen realitätsnaher komplexer Probleme auftritt. Die Ergebnisse der Feldforschung liefern Anhaltspunkte hierfür (Comer 1995; Earley 1989; Gabrenya et al. 1983, 1985; Williams, Nida, Baca und Latané 1989). 
Arbeitsbedingung. Die Manipulation der Arbeitsbedingung sieht entweder eine koaktive oder eine kollektive Verantwortlichkeit für das Gesamtergebnis vor. Damit soll überprüft werden, ob beim Lösen komplexer Probleme unter koaktiven oder unter kollektiven Bedingungen bessere Leistungen erzielt werden. Da sich die Unterscheidung zwischen den beiden Arbeitsbedingungen auf die Transparenz hinsichtlich der individuellen und kollektiven Leistungen bezieht und zumindest im ersten Experiment keine Interaktion mit den Mitspielern möglich ist, lässt sich die zu erwartende geringere Anzahl an Kommandos unter der kollektiven Arbeitsbedingung nicht auf ein verändertes Interaktionsverhalten zurückführen.

Komplexität. Die Komplexität des Problems soll in den zwei Stufen „einfach“ und „komplex“ erfolgen. Damit lassen sich Auswirkungen des Komplexitätsniveaus auf die Motivation und auf die Leistungen der Teilnehmenden überprüfen.

Für die abhängigen Variablen Motivation und Leistung lassen sich mit Hilfe des Szenarios Networked Fire Chief unabhängige Werte für beide Größen erheben. Das Programm erlaubt es, die individuelle und die kollektive Leistung der Spieler in Form von Prozentsätzen geretteter Fläche bzw. Objekte zu ermitteln. Der im Anschluss an die Simulationsbearbeitung ausgegebene „statistics report" liefert neben diesen allgemeinen Leistungsindikatoren, die Aufschluss über die Leistungen der jeweiligen Einzelspieler wie auch der Gruppe insgesamt geben, auch Indikatoren für das individuelle Eingriffsverhalten als Maß für die Anstrengung. Die Motivation bzw. Anstrengung im Umgang mit der Simulation wird über dieses Eingriffsverhalten operationalisiert und bezieht sich konkret auf die Anzahl gegebener Kommandos. Die Wahl der Anstrengung als Motivationsindikator findet sich in der gesamten Forschung zum sozialen Faulenzen: Auf der Grundlage des beobachteten Verhaltens wird auf die dahinter liegenden motivationalen Strukturen geschlossen. Eine geringere Anstrengung in kollektiven gegenüber koaktiven Situationen wird demnach auf die Motivationsverluste in kollektiven Situationen zurückgeführt (Karau und Williams 1993, 1995; Shepperd 1993).

Insgesamt erlaubt das Szenario Networked Fire Chief die Bestimmung der Werte für Anstrengung und Leistung auf der Grundlage unabhängiger Daten. Dies war für einen Großteil der Aufgabenstellungen, die bislang der Forschung im Bereich soziales Faulenzen zu Grunde lagen, nicht möglich.

\section{Hypothesen}

Auf der Grundlage der dargelegten Modellvorstellungen wurden Hypothesen formuliert, die Aussagen über die Zusammenhänge zwischen den unabhängigen, den intervenierenden und den abhängigen Variablen für die jeweiligen Treatments erlauben. Die unabhängigen Variablen ergeben sich aus dem Versuchsaufbau, wohingegen die intervenierenden Variablen auf die theoretischen Annahmen des CEM zurückzuführen sind. Insgesamt ist davon auszugehen, dass sich Personen beim Lösen komplexer Probleme in kollektiven Situationen (Realgruppen) in geringerem Maße anstrengen als Personen in koaktiven Situationen (Nominalgruppen).

Das Auftreten von sozialem Faulenzen lässt sich mit Hilfe des CEM (Karau und 
Williams 1993) dadurch erklären, dass die wahrgenommene Instrumentalität in kollektiven Situationen geringer als in koaktiven Situationen ist. Eine hohe Anstrengung ist dem Modell nach auch davon abhängig, dass die Akteure die Handlungsfolgen im Sinne der Ergebnisse hoch bewerten müssen. Auf Grund der unmittelbaren Erreichbarkeit der Ergebnisse unter koaktiven Arbeitsbedingungen ist davon auszugehen, dass diese in solchen Situationen auch höher bewertet werden als in kollektiven Situationen.

Im Falle des einfachen Problems erwarten wir, dass Motivation und Leistung in einem positiven linearen Verhältnis zueinander stehen werden, wohingegen im Falle des komplexen Problems ein inverses Verhältnis von Motivation und Leistung erwartet wird. Demnach führt beim Lösen des einfachen Problems die höhere Anstrengung in koaktiven Gruppen zu besseren Leistungen, wohingegen beim Lösen des komplexen Problems die höhere Anstrengung dieser Gruppen zu schlechteren Leistungen führt. Begründen lässt sich dies vor allem durch triebtheoretische Annahmen. Ihnen zufolge führt die individuelle Zurechenbarkeit von Leistungen zu einer Trieberhöhung, die eine Anwendung bereits ausgeprägter Verhaltensmuster bei der Problemlösung begünstigt (Jackson und Williams 1985). Beim Bearbeiten einfacher Probleme führe dies zu problemadäquatem Verhalten, weshalb die Leistung in koaktiven Situationen höher ist als in Situationen mit geringem Triebniveau. Beim Bearbeiten des komplexen Problems steigt in koaktiven Situationen hingegen die Fehlerhäufigkeit. Trieberhöhung geht in diesem Falle mit Leistungseinbußen einher. Folglich werden in koaktiven Situationen schlechtere Leistungen erzielt als in kollektiven Situationen.

Auch die Theorien des Selbst stützen diese Erklärungen für das inverse Verhältnis von Anstrengung und Leistung. Der Theorie des Selbstbewusstseins zufolge sind die Personen beim Lösen einfacher Probleme eher in der Lage, einen Leistungsstandard zu erzielen und - sofern dieser ihrem Anspruchsniveau Stand hält - in stärkerem Maße mit ihren Leistungen zufrieden (Duval und Wicklund 1972, 1973; Wicklund 1975). Beim Lösen des komplexen Problems wird dieser Leistungsstandard nicht so einfach erzielt, weshalb sich Personen umso mehr anstrengen. Diese relativ höhere Anstrengung kann beim Lösen dieser Probleme aber zu suboptimalen Leistungen führen. Der Theorie der Selbstdarstellung folgend gelingt den Personen beim Lösen einfacher Probleme eine bessere Selbstdarstellung, wohingegen dies beim Lösen schwieriger Probleme nicht der Fall ist (Schlenker und Leary 1982). In solchen Situationen kann es daher auch zu Stress und kognitiven Störungen kommen, was ein problemadäquates Verhalten nicht wahrscheinlich werden lässt.

\section{Versuchspersonen}

Mit Hilfe des Programms G*Power von Buchner, Faul und Erdfelder (1992; siehe auch Erdfelder et al. 1996) wurde eine a-priori Poweranalyse durchgeführt, aus der sich eine Stichprobengröße von $\mathrm{n}=30$ für den einfachen Mittelwertvergleich zweier Stichproben ergibt. Dies gilt für eine $\mathrm{t}$-Testsituation bei einer liberalen Irrtumswahrscheinlichkeit von $\alpha=0.10$ und $\beta=0.20$ und bei der Erwartung einer großen Effektstärke ( $\mathrm{d}=0.80$ sensu Cohen 1988).

Eine zweite a-priori Poweranalyse wurde für die Varianzanalyse (ANOVA) durchge- 
führt, aus der sich eine Stichprobengröße $\mathrm{n}=52$ ergibt. Für eine F-Testsituation gilt dies bei einer Irrtumswahrscheinlichkeit von $\alpha=0.05$ und $\beta=0.20$ und bei der Erwartung einer großen Effektstärke ( $f=0.40$ sensu Cohen 1988).

Für die Berechnung statistischer Zusammenhänge ergibt sich demnach eine Stichprobengröße von 60 Personen. Da bei der Durchführung der Untersuchung vorgesehen ist, dass die Probanden jeweils nur an einem Experiment teilnehmen, ergab sich eine Gesamtstichprobenzahl von 120 Personen.

An Experiment 1 haben 60 Personen (38 Frauen und 22 Männer) im Alter von 18 bis 49 Jahren teilgenommen. Die Probanden wurden in Dreiergruppen einbestellt und nach dem Zufallsprinzip einer Treatmentbedingung zugewiesen. Die Teilnahme war mit einem zeitlichen Aufwand von drei Stunden verbunden und wurde mit einer Aufwandsentschädigung vergütet.

\section{Versuchsdurchführung}

Die Versuchsdurchführung erfolgte in drei Abschnitten: einer Einführung, einer Trainings- und einer Versuchsphase. Die Teilnehmenden wurden mit Hilfe einer Demoversion in das Programm eingeführt. Bereits in der Einführung wurde den Teilnehmenden kollektiver Gruppen mitgeteilt, es gehe um das Erbringen einer Gruppenleistung, wohingegen den Teilnehmenden koaktiver Gruppen mitgeteilt wurde, das Programm zeichne die Leistungen der drei Flächen getrennt auf und ihre Aufgabe bestünde darin, in der ihnen zugeteilten Fläche die Feuer möglichst gut zu bekämpfen.

Nach der Einführung erfolgten drei Probedurchläufe. Diese dienten einerseits dazu, die Probanden aktiv mit dem Programm vertraut zu machen, andererseits dazu, die individuellen Leistungsvoraussetzungen der Probanden zu erheben. Nach den Trainingsdurchgängen finden die Versuchsdurchgänge statt. Im Anschluss an jeden der drei Versuchsdurchgänge wurde den Teilnehmenden ein Fragebogen vorgelegt. Ziel dieser Befragung war es, Aufschluss darüber zu gewinnen, inwiefern die wahrgenommene Instrumentalität und die Bewertung der Problemstellung die Motivation und Leistung der Probanden bei der Bearbeitung der Problemstellung beeinflussen. Darauf wird jedoch in dieser Arbeit nicht näher eingegangen (ausführlicher dazu siehe Feuchter 2001).

\section{Ergebnisse}

Zunächst werden die Ergebnisse aus Experiment 1 dargestellt, in dem die Versuchspersonen nur auf ihrem eigenen Feld agieren konnten. Danach werden die Befunde aus Experiment 2 berichtet, in dem jede Person auf allen Feldern agieren konnte. 


\section{Ergebnisse von Experiment 1}

a) Anstrengung. Zunächst zum Eingriffsverhalten, das die Anstrengung reflektiert. Insgesamt kann ein signifikanter Anstrengungs- bzw. Motivationsverlust nachgewiesen werden. Die Manipulation der Situationsdefinition hat das Eingriffsverhalten der Probanden bei der Steuerung des Systems stark beeinflusst: Erwartungsgemäß werden in der koaktiven Situation signifikant mehr Kommandos ( $M=111,70 ; S D=19,38)$ abgegeben als in der kollektiven Situation $(M=99,86 ; S D=14,77 ; F(1,56)=7,61$, $\mathrm{p}<0.01)$.

Ein Mittelwertsvergleich der Gruppen mit unterschiedlichem Komplexitätsniveau ergibt für das komplexe Szenario einen signifikanten Einfluss der Situationsdefinition auf die Anstrengung. Die Probanden koaktiver Gruppen $(M=107,67 ; S D=20,94)$ erbringen signifikant mehr Kommandos als die Probanden in der Vergleichsgruppe $(M=93,42 ; S D=15,58 ; F(1,28)=4,466, p<0.05)$. Für die einfache Problemstellung ist die Differenz zwischen der Anzahl an Kommandos in den koaktiven $(M=115,73$; $\mathrm{SD}=17,44)$ und den kollektiven Gruppen $(\mathrm{M}=106,29 ; \mathrm{SD}=10,98 ; \mathrm{F}(1,28)=3,15$, n.s.) zwar gegeben, wenn auch nicht signifikant.

Tabelle 1: Mittelwerte (M) und Standardabweichungen (SD) der abhängigen Variable „Anstrengung“ in Abhängigkeit von Komplexitätsniveau (einfach oder komplex) und Situationsdefinition (koaktiv oder kollektiv) aus Experiment 1 $(\mathrm{N}=60)$

\begin{tabular}{lccc}
\hline & Koaktive Gruppen & Kollektive Gruppen & $M_{\text {gesamt }}$ \\
\hline Einfache Problemstellung & 115,73 & 106,29 & 111 \\
$\mathrm{M}$ & 15 & 15 & 30 \\
$\mathrm{~N}$ & 17,44 & 10,98 & \\
$\mathrm{SD}$ & & & 100,6 \\
Komplexe Problemstellung & 107,67 & 93,42 & 30 \\
$\mathrm{M}$ & 15 & 15 & \\
$\mathrm{~N}$ & 20,94 & 15,58 & \\
$\mathrm{SD}$ & 111,7 & 99,9 & \\
\hline $\mathrm{M}_{\text {gesamt }}$ & 30 & 30 & \\
\hline
\end{tabular}

b) Leistung. Im Unterschied zum Einfluss der Situationsdefinition auf die Anstrengung gibt es keine Differenz in der Leistung zwischen koaktiven $(\mathrm{M}=80,28 ; \mathrm{SD}=9,6)$ und kollektiven Gruppen $(\mathrm{M}=82,12 ; \mathrm{SD}=6,9 ; \mathrm{F}(1,56)=2,08$, n.s). Es lässt sich kein Haupteffekt der Situationsdefinition auf die Leistung nachweisen. Betrachtet man den Einfluss des Interaktionseffektes von Situationsdefinition und Komplexitätsniveau, so trägt dieser jedoch maßgeblich zur Aufklärung der Varianz bei $(\mathrm{F}(1,56)=6,16$, $\mathrm{p}<0.02)$.

Eine getrennte Betrachtung der einfachen und der komplexen Problemstellung lässt für das komplexe Szenario einen signifikanten Unterschied zwischen den Leistungen koaktiver $(M=72,2 ; S D=5,52)$ und kollektiver Gruppen $(M=77,22 ; S D=4,72$; $\mathrm{F}(1,28)=7,172, \mathrm{p}<0.02)$ erkennbar werden. Für das einfache Szenario ergibt sich hingegen nahezu keine Leistungsdifferenz zwischen der koaktiven $(M=88,36$; 
Abbildung 3: Anstrengung und Leistung a) im einfachen und b) im komplexen Szenario ohne Interaktionsmöglichkeit (Experiment 1)

Abbildung $3 a$

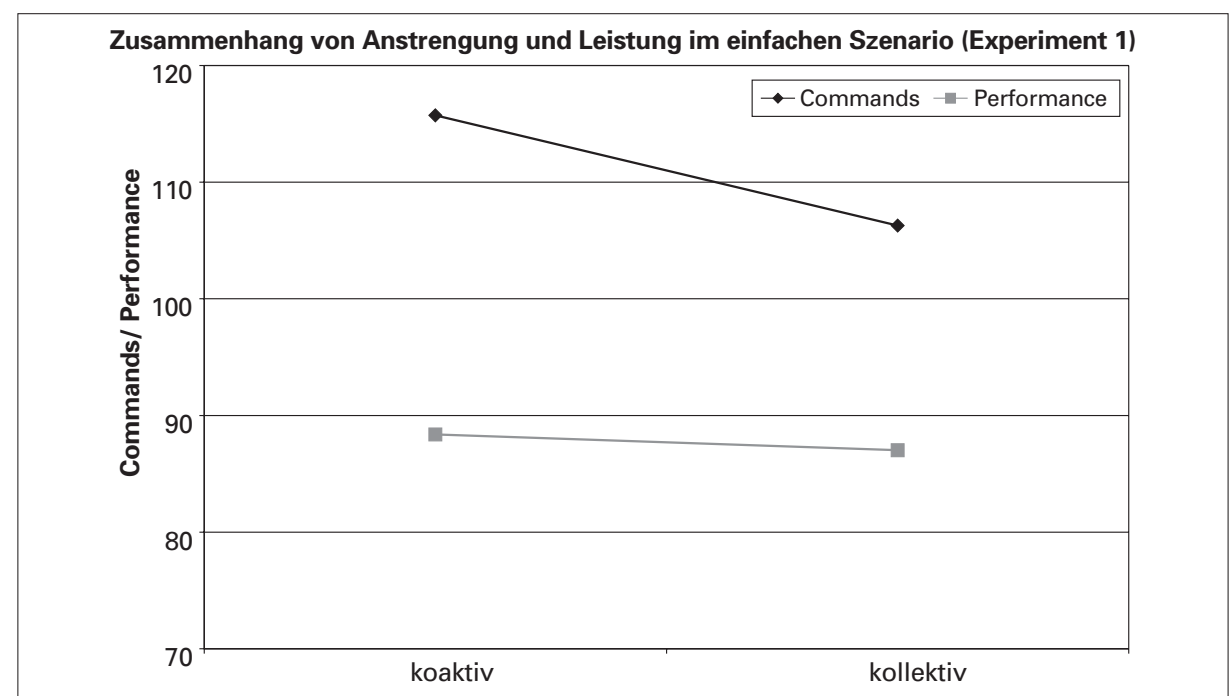

Abbildung $3 b$

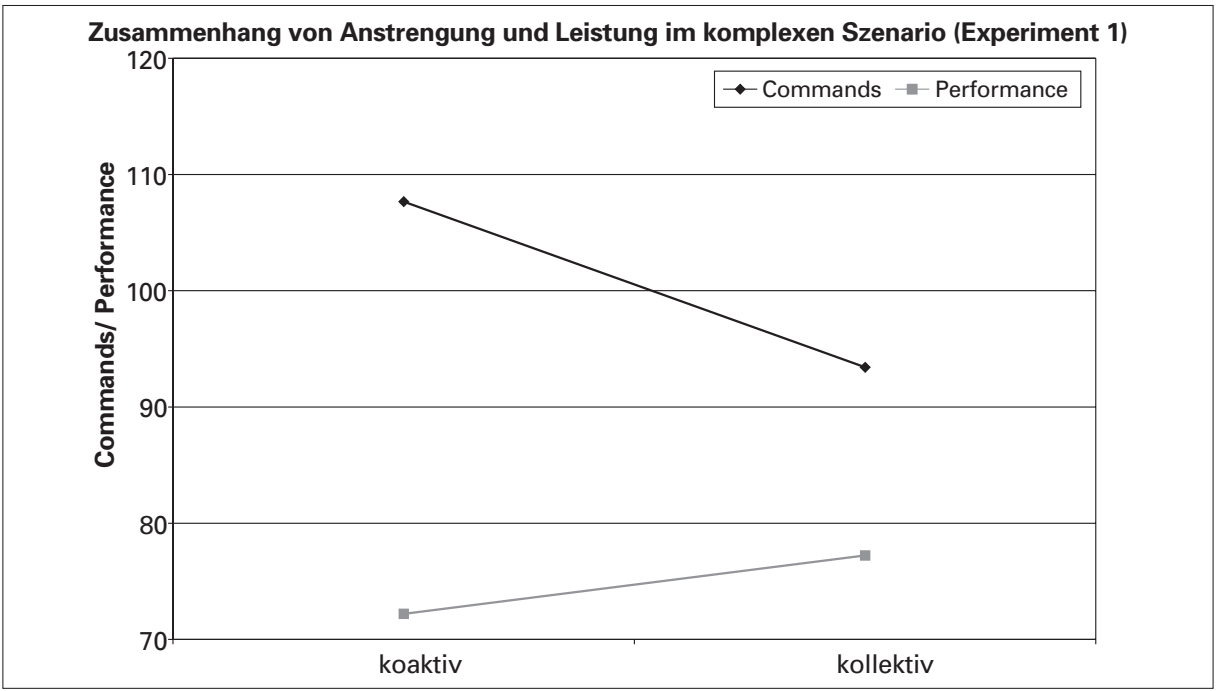

$\mathrm{SD}=4,55)$ und der kollektiven Situation $(\mathrm{M}=87,02 ; \mathrm{SD}=4,99 ; \mathrm{F}(1,28)=0,585$, n.s.). 


\section{Interpretation von Experiment 1}

Vor dem Hintergrund der Hypothesenentscheidung zeigt sich, dass im ersten Experiment sowohl die Manipulation des Komplexitätsniveaus der Problemstellung als auch die Manipulation der Arbeitsbedingung einen Einfluss auf die beiden abhängigen Variablen Motivation und Leistung haben. Insgesamt unterstützen die Ergebnisse des ersten Experiments die Annahme, dass auch beim Lösen komplexer Probleme soziales Faulenzen auftritt. Sowohl für die einfache als auch für die komplexe Problemstellung konnte eine Differenz der jeweiligen Anstrengungswerte zwischen den beiden Vergleichsgruppen nachgewiesen werden. Diesen Ergebnissen folgend stellt das zu Grunde liegende komplexe Problem Networked Fire Chief ein geeignetes Szenario zum Nachweis des Auftretens von sozialem Faulenzen unter kollektiven Arbeitsbedingungen dar. Darüber hinaus war es auf Grund der Unterscheidung zwischen Anstrengung und Leistung möglich, einen inversen Effekt von Anstrengung und Leistung beim Lösen des komplexen Szenarios auszuweisen. Wie sich gezeigt hat, erzielten die Gruppen, die das komplexe Problem unter der kollektiven Bedingung bearbeitet haben, trotz geringerer Anstrengung eine signifikant höhere Leistung als die Vergleichsgruppen. Umgekehrt hat sich auch die Erwartung bestätigt, dass die höhere Anstrengung der Mitglieder koaktiver Gruppen, die das einfache Szenario bearbeitet haben, einen positiven Einfluss auf die Leistung hat. Daher lässt sich vor dem Hintergrund der Ergebnisse des ersten Experiments festhalten, dass soziales Faulenzen beim Lösen eines komplexen Problems positiv zu bewerten ist. Ob dies auch gilt, wenn die Komplexität durch Einführung weiterer Interaktionsmöglichkeiten gesteigert wird, zeigt das nachfolgend beschriebene Experiment 2.

\section{Ergebnisse von Experiment 2}

Im Unterschied zum Experiment 1, in dem die Probanden nur auf der ihnen jeweils zugewiesenen Fläche die Feuer bekämpfen konnten, war es ihnen in Experiment 2 auch möglich, die Feuer auf allen drei Feldern zu bekämpfen, also auch bei den zwei Mitspielern einzugreifen. Auf Grund dieser Interaktionsmöglichkeiten konnten in Experiment 2 schlechtere Leistungen einzelner Spieler durch bessere Leistungen der Mitspieler ausgeglichen und entsprechend kompensiert werden. Dies entspricht in der Steinerschen Taxonomie einer konjunktiven Aufgabenstellung (vgl. Steiner 1972). Die potenziellen Interaktionsmöglichkeiten bedingen eine Komplexitätssteigerung der sozialen Situation, die wegen der gegenseitigen Abhängigkeit der Personen als eine Situation doppelter Kontingenz (vgl. Parsons 1951) aufzufassen ist, da die Problemlöser sich in ihrem Handeln gegenseitig beeinflussen können.

Vor dem Hintergrund dieser Modifikation war zu erwarten, dass soziales Faulenzen im zweiten Experiment noch in deutlicherem Maße auftreten wird als im ersten Experiment, da die vernetzte Problemlösung, bedingt durch die grenzüberschreitenden Interventionsmöglichkeiten, die individuelle Zurechenbarkeit der Leistungen faktisch unmöglich werden lässt.

Aus der Perspektive der Theorie des kollektiven Handelns muss vermutet werden, 
dass die Motivationsverluste mit der Steigerung der Nichtzurechenbarkeit noch zunehmen werden. Im Übrigen gilt für das zweite Experiment, wie auch schon für das erste, dass die Bedingungen, welche zu einer Abschwächung oder Lösung des Trittbrettfahrerproblems führen können, in den Versuchsbedingungen nicht gegeben sind. Zwar ist eine Iteration durch wiederholte Durchgänge gegeben, aber diese „löst“ das Motivationsproblem nur, wenn sie nicht endlich ist (vgl. Axelrod 1991). Auch andere Mechanismen, welche Motivationsverluste der Theorie der rationalen Wahl nach einschränken könnten, wie die Kommunikation, das Vertrauen oder selektive Anreize können angesichts des Versuchsaufbaus nur in rudimentärer Form zum Tragen kommen.

Die Komplexitätssteigerung der sozialen Situation könnte ferner dazu führen, dass es bereits unter der einfachen Problemstellung zu einem inversen Effekt von Anstrengung und Leistung kommen kann. Das Auftreten eines solchen Effekts ist allein schon deswegen wahrscheinlich, da die Komplexität der sozialen Situation - bedingt durch die Interaktionsmöglichkeiten im zweiten Experiment - mit der Komplexität des Problems konfundiert und beide zusammen eine Komplexitätssteigerung bewirken.

Ansonsten wurden die Versuchsbedingungen von Experiment 1 auch in Experiment 2 beibehalten, d.h. der Einfluss unterschiedlicher Arbeitsbedingungen und unterschiedlicher Komplexitätsniveaus der Problemstellung auf Anstrengung und Leistung untersucht. Hierfür wurde der bereits aus Experiment 1 bekannte 2 (koaktive vs. kollektive Situation) $\times 2$ (einfaches vs. komplexes Problem) faktorielle Versuchsplan (Within-Subject-Design) erneut verwendet.

An Experiment 2 nahmen insgesamt 60 Personen (52 Frauen und 8 Männer) im Alter von 18 bis 30 Jahren teil, die nicht bereits zuvor an Experiment 1 teilgenommen hatten. Wie in Experiment 1 wurden auch hier die Probanden wieder in Dreiergruppen einbestellt und zufällig einer der vier Treatmentbedingungen zugeteilt.

Ergebnisse. Zunächst werden die Befunde in Hinblick auf die Anstrengung, dann bezogen auf die erbrachte Leistung berichtet.

a) Anstrengung. Erneut kann insgesamt ein signifikanter Anstrengungsverlust ausgemacht werden. Die Probanden der Gruppen, die unter der kollektiven Arbeitsbe-

Tabelle 2: Mittelwerte (M) und Standardabweichungen (SD) der abhängigen Variable "Anstrengung" in Abhängigkeit von Komplexitätsniveau (einfach oder komplex) und Situationsdefinition (koaktiv oder kollektiv) aus Experiment 2 $(\mathrm{N}=60)$

\begin{tabular}{lccc}
\hline & Koaktive Gruppen & Kollektive Gruppen & M $_{\text {gesamt }}$ \\
\hline Einfache Problemstellung & 109,84 & 103,84 & 106,8 \\
$\mathrm{M}$ & 15 & 15 & 30 \\
$\mathrm{~N}$ & 13,34 & 18,31 & \\
$\mathrm{SD}$ & & & \\
Komplexe Problemstellung & 105,84 & 96,84 & 101,3 \\
$\mathrm{M}$ & 15 & 15 & 30 \\
$\mathrm{~N}$ & 11,18 & 7,92 & \\
$\mathrm{SD}$ & 107,8 & 100,3 & 30 \\
\hline $\mathrm{M}_{\text {gesamt }}$ & 30 & 30,3 & \\
\hline
\end{tabular}


Abbildung 4: Anstrengung und Leistung a) im einfachen und b) im komplexen Szenario mit Interaktionsmöglichkeit (Experiment 2)

Abbbildung $4 a$

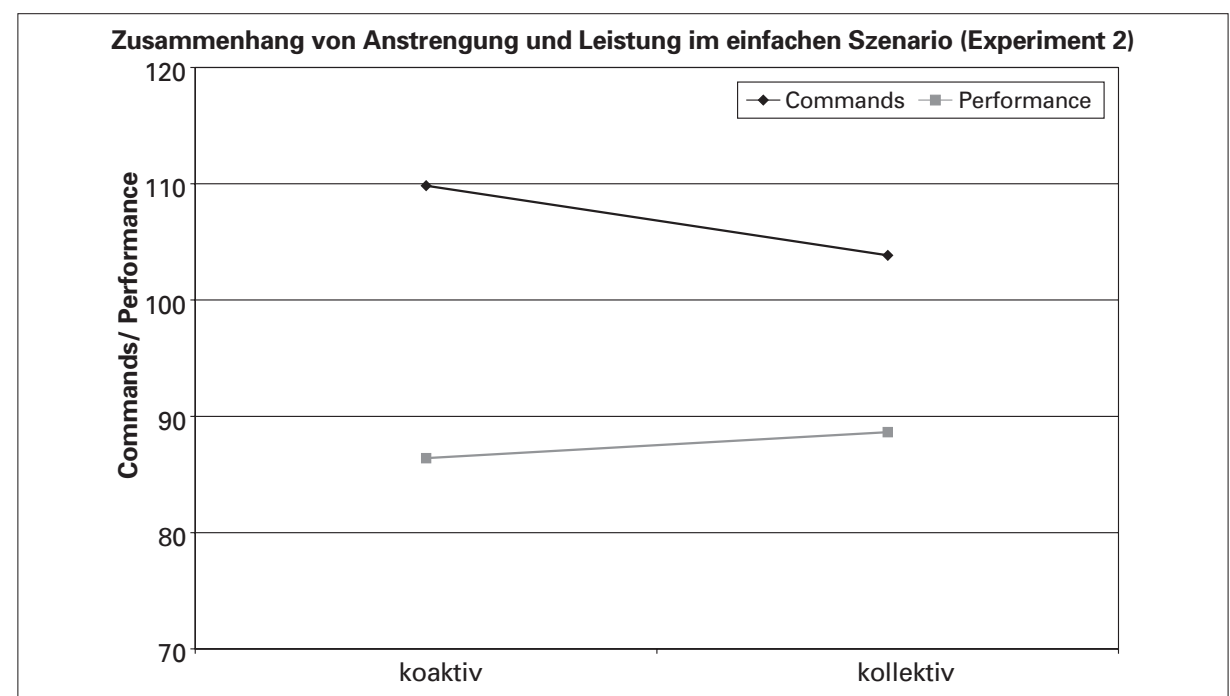

Abbbildung $4 b$

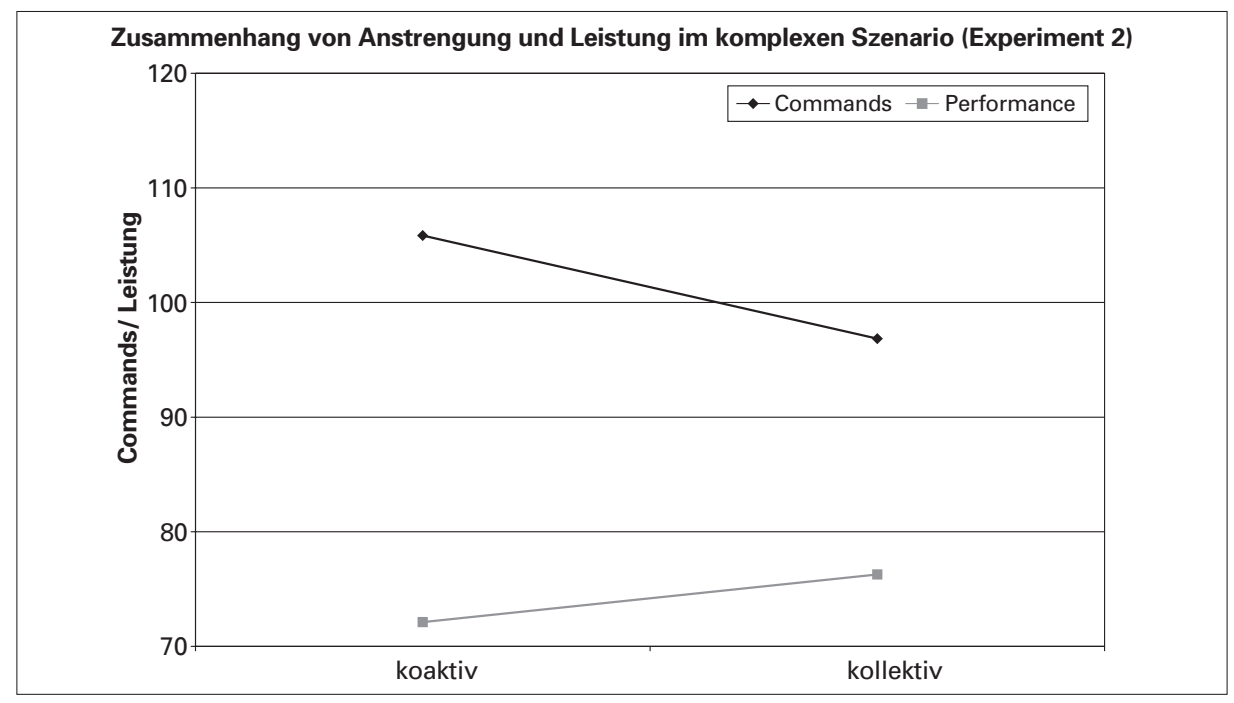

dingung das Problem gelöst haben, gaben signifikant weniger Kommandos ab $(M=100,34 ; S D=14,31)$ als die Probanden in den Vergleichsgruppen $(M=107,84$; $\mathrm{SD}=12,26 ; \mathrm{F}(1,56)=4,81, \mathrm{p}<0,05)$. Der Einfluss des Komplexitätsniveaus auf die Anstrengung ist hingegen nicht signifikant $(\mathrm{F}(1,56)=2,59$, n.s. $)$. 
Ein Mittelwertsvergleich der Kommandos zwischen den Gruppen mit unterschiedlichen Komplexitätsniveaus zeigt für die komplexe Problemstellung einen signifikanten Unterschied zwischen koaktiven und kollektiven Gruppen. Probanden koaktiver Gruppen $(\mathrm{M}=105,84 ; \mathrm{SD}=11,18)$ haben signifikant mehr Kommandos abgegeben als diejenigen kollektiver Gruppen $(\mathrm{M}=96,84 ; \mathrm{SD}=7,92 ; \mathrm{F}(1,28)=6,47, \mathrm{p}<0,05)$.

b) Leistung. Betrachtet man die Leistung, so lassen sich für die beiden unabhängigen Variablen Arbeitsbedingung und Komplexitätsniveau der Problemstellung jeweils Haupteffekte ausmachen. Die Situationsdefinition beeinflusst demnach die Leistungsdifferenz zwischen koaktiven $(\mathrm{M}=79,27 ; \mathrm{SD}=8,77)$ und kollektiven Gruppen $(\mathrm{M}=82,46 ; \mathrm{SD}=8,2 ; \mathrm{F}(1,56)=5,71, \mathrm{p}<0,01)$. In ähnlicher Weise beeinflusst das Komplexitätsniveau die Leistungen der unterschiedlichen Gruppen $(F(1,56)=6,05$, $\mathrm{p}<0,01)$. Eine nach Komplexitätsniveaus getrennte Analyse ergibt für beide Komplexitätsniveaus keine signifikante Differenz zwischen der kollektiven und der koaktiven Situation. In der einfachen Problemsituation unterscheiden sich die Werte zwischen koaktiven $(M=86,42 ; S D=4,02)$ und kollektiven Gruppen $(M=88,64 ; S D=4,34)$ nicht $(\mathrm{F}(1,28)=2,12$, n.s). In der komplexen Problemsituation findet sich erwartungsgemäß ein deutlicherer Unterschied zwischen koaktiven $(M=72,11 ; S D=5,52)$ und kollektiven Gruppen $(M=76,27 ; S D=6,2 ; F(1,28)=3,41, p<0,07)$.

\section{Interpretation von Experiment 2}

Auch in Experiment 2 konnte somit unter der kollektiven Bedingung das Auftreten von sozialem Faulenzen nachgewiesen werden. Entsprechend den theoretischen Erwartungen ist hier der inverse Effekt von Anstrengung und Leistung im Falle des einfachen Problems eingetreten (vgl. Abbildung 4). Die Anstrengung in der koaktiven $(\mathrm{M}=109,84 ; \mathrm{SD}=13,34)$ liegt erwartungsgemäß über jener in der kollektiven Situation $(\mathrm{M}=103,84 ; \mathrm{SD}=18,31 ; \mathrm{F}(1,28)=1,05$, n.s.), gleichzeitig findet sich im Hinblick auf die Leistung für die einfache Problemstellung - obgleich nicht signifikant eine höhere Leistung in der kollektiven $(M=88,64 ; S D=4,34)$ als in der koaktiven Situation $(\mathrm{M}=86,42 ; \mathrm{SD}=4,02 ; \mathrm{F}(1,28)=2,12$, n.s. $)$.

Dieses Resultat ist dadurch zu erklären, dass die Komplexitätssteigerung der sozialen Situation zu denselben Effekten wie die Komplexitätssteigerung des Problems führt und die Steigerung der Komplexität der sozialen Situation ausschlaggebend dafür ist, dass sich im zweiten Experiment bereits für die einfache Problemstellung ein inverses Verhältnis von Anstrengung und Leistung abzeichnet.

Für das komplexe Problem zeichnet sich ebenfalls ein inverses Verhältnis von Anstrengung und Leistung ab. Probanden koaktiver Gruppen haben signifikant mehr Kommandos abgegeben $(\mathrm{M}=105,84 ; \mathrm{SD}=11,18)$ als diejenigen kollektiver Gruppen $(\mathrm{M}=96,84 ; \mathrm{SD}=7,92 ; \mathrm{F}(1,28)=6,473, \mathrm{p}<0,05)$ und es liegt ein deutlicher Unterschied zwischen den Leistungen koaktiver $(\mathrm{M}=72,11 ; \mathrm{SD}=5,52)$ und kollektiver Gruppen vor $(\mathrm{M}=76,27 ; \mathrm{SD}=6,2 ; \mathrm{F}(1,28)=3,41, \mathrm{p}<0,07)$.

Die Resultate des zweiten Experiments belegen erneut die Annahme, dass beim Lösen komplexer Probleme soziales Faulenzen auftritt und Teilnehmende unter kollekti- 
ven Arbeitsbedingungen in geringerem Maße motiviert sind als jene unter koaktiven Arbeitsbedingungen. ${ }^{3}$ Im Unterschied zum ersten wurde im zweiten Experiment eine Komplexitätssteigerung der sozialen Situation vorgenommen, um zu überprüfen, ob diese dieselben Auswirkungen auf das Verhältnis von Motivation und Leistung hat, wie die Komplexitätssteigerung des Problems. Da sich dieser Effekt nachweisen ließ, ist es auch nicht erstaunlich, dass das Ergebnis des zweiten Experiments von jenem des ersten abweicht. Wie sich gezeigt hat, erzielten im zweiten Experiment kollektive Gruppen auch beim Bearbeiten des einfachen Problems bessere Leistungen als koaktive Gruppen, wohingegen im ersten Experiment die koaktiven Gruppen beim Lösen des einfachen Problems höhere Leistungen als die Vergleichsgruppen erbrachten.

Zusammenfassend liegt im zweiten Experiment wie im ersten für die komplexe Problemstellung ein inverser Effekt von Anstrengung und Leistung vor. Damit kann auch für Experiment 2 die These bestätigt werden, dass beim Lösen des komplexeren Problems die höhere Anstrengung zu schlechteren Leistungen führt und soziales Faulenzen eine bessere Bewältigung des Problems in Aussicht stellt.

\section{Schluss}

Die vorliegende Untersuchung hat den Nachweis erbracht, dass beim Lösen komplexer Probleme unter kollektiven Arbeitsbedingungen soziales Faulenzen auftritt. Wie sich gezeigt hat, wirkt sich dieser Motivationsverlust bei gesteigerter Komplexität des Problems einerseits und der sozialen Situation andererseits positiv auf die Güte der individuellen und der kollektiven Problemlösung aus. Auf der Grundlage des wert-erwartungstheoretischen CEM (Karau und Williams 1993) wurden die Einflussfaktoren auf die Motivation und die Leistung beim Lösen komplexer Probleme formuliert. Die der Untersuchung zu Grunde liegende Analyse gewährt zudem Aufschluss darüber, wodurch sich das zunächst paradox erscheinende Verhältnis erklären lässt, dass geringere Motivation beim Lösen eines einfachen Problems zu schlechteren Leistungen, beim Lösen eines komplexen Problems dagegen zu besseren Leistungen führt.

Das Auftreten von sozialem Faulenzen konnte - wenn auch nur bedingt - entsprechend den Erwartungen des CEM auf das geringere Maß an wahrgenommener Instrumentalität und auf die geringere Bewertung der Handlungskonsequenzen unter kollektiven Bedingungen zurückgeführt werden. Die Annahmen des CEM ließen sich somit bestätigen. Determiniert wurde der Grad der Anstrengung aber auch durch weitere Faktoren. In der Studie von Feuchter (2001) wurde gezeigt, dass nicht-kognitive Faktoren wie Stress, Erregung und irrelevante Informationsverarbeitung unter den koaktiven Arbeitsbedingungen in weitaus höherem Maße ausgeprägt waren als unter den kollektiven Bedingungen. Beim Lösen komplexer Probleme unter koaktiven Arbeitsbedingungen führten diese höheren Werte zu Leistungseinbußen gegenüber den geringeren Werten unter den kollektiven Arbeitsbedingungen. Entsprechend wurde das CEM zur

3 Es ist nicht auszuschließen, dass die geringere Zahl an Kommandos im zweiten Experiment auf eine stärkere Konzentration auf die Interventionen der Mitspieler gründet. Standardmäßig waren deren Aktivitäten aber nur ausschnittsweise zu sehen, so dass diesem Einfluss auf die Motivation kein zu hoher Wert beizumessen ist. 
Analyse der Auswirkungen von Motivationsverlusten auf die Leistung beim Lösen komplexer Probleme durch die Faktoren Stress, Erregung und irrelevante Informationsverarbeitung ergänzt (Feuchter 2001). Diese Faktoren, die sich in der Instrumentalitätstheorie von Karau und Williams (1993, 1995, 1997, 2000) nicht finden lassen, tragen zur Erklärung der Differenz zwischen den Anstrengungswerten koaktiver und kollektiver Gruppen bei.

Die Studie hat nachgewiesen, dass bei steigender Komplexität der Problemstellung ein zunächst paradox erscheinendes Verhältnis zwischen Motivation und Leistung auftritt, indem geringere Motivation zu besseren Leistungen führt. Bereits Bartis et al. (1988) sowie Jackson und Williams (1985) konnten dieses Paradox für schwierige Aufgaben nachweisen. Was in ihrer Analyse aber ausblieb, war eine zufrieden stellende Erklärung für das Verhältnis von Motivation und Leistung. Vor dem Hintergrund dieses Forschungsdesiderats wurde in der eigenen Untersuchung ein Modell entwickelt, welches einen Erklärungsrahmen für das Auftreten dieses zunächst paradox erscheinenden Phänomens abgibt (ausführlicher siehe Feuchter 2001). Bei komplexen Problemen folgt aus erhöhter Anstrengung auch eine Erhöhung des Stressniveaus, was zu einer gesteigerten Fehlerhäufigkeit führt. Bei einfachen Problemen führt die Erhöhung des Stressniveaus nicht in dem Maße zu Fehlern und damit auch zu keiner verringerten Leistung. Unseres Erachtens wäre die Durchführung weiterer Studien wünschenswert, um die ökologische Validität des inversen Verhältnisses von Motivation und Leistung belegen zu können.

Der Nachweis des positiven Einflusses einer geringeren Motivation auf die Leistung für das Lösen von komplexen Problemen hat auch Konsequenzen für die Aussagen seitens der Vertreter der sozialpsychologischen Forschung, die soziales Faulenzen im Allgemeinen negativ bewerten: „It is a ,disease“ in that it has negative consequences for individuals, social institutions, and societies. Social loafing results in a reduction in human efficiency, which leads to lowered profits and lowered benefits for all. It is social ${ }^{6}$ in that it results from the presence or actions of other people" (Latané et al. 1979: 831). Im Lichte der Ergebnisse der vorliegenden Studie würde soziales Faulenzen gerade keine soziale Krankheit, sondern vielmehr eine Chance zur Leistungssteigerung in bestimmten Situationen darstellen.

\section{Literatur}

Arnscheid, Rüdiger, Michael Diehl und Wolfgang Stroebe, 1997: Motivationsverluste in Gruppen: Ein empirischer Test einer theoretischen Integration. Zeitschrift für Sozialpsychologie 28: 241-250.

Axelrod, Robert, 1984: The Evolution of Cooperation. New York: Basic Books.

Bartis, Scott, Kate Szymanski und Stephen G. Harkins, 1988: Evaluation and Performance: A Twoedged Knife. Personality and Social Psychology Bulletin 14: 242-251.

Brickner, Mary A., Stephen G. Harkins und Thomas M. Ostrom, 1986: Effects of Personal Involvement: Thought-provoking Implications for Social Loafing. Journal of Personality and Social Psychology 51: 763-770.

Brown, Rupert, 2000: Group Processes: Dynamics within and between Groups. Oxford, UK: Blackwell. 
Buchner, Axel, Franz Faul und Edgar Erdfelder, 1992: G•Power: A priori, Post-hoc and Compromise Power Analyses for the Macintosh [Computer program]. Bonn, FRG: Psychologisches Institut der Universität Bonn.

Cohen, Jacob, 1988: Statistical Power Analysis for the Behavioral Sciences (2nd ed.). Hillsdale, NJ: Lawrence Erlbaum Associates.

Comer, Debra R., 1995: A Model of Social Loafing in Real Work Groups. Human Relations 48: 647-667.

Duval, Shelly, und Robert A. Wicklund, 1972: A Theory of Objective Self-awareness. New York: Academic Press.

Duval, Shelly, und Robert A. Wicklund, 1973: Effects of Objective Self-awareness on Attribution of Causality. Journal of Experimental Social Psychology 9: 17-31.

Earley, P. Christopher, 1989: Social Loafing and Collectivism: A Comparison of the United States and the People's Republic of China. Administrative Science Quarterly 34: 565-581.

Erdfelder, Edgar, Franz Faul und Axel Buchner, 1996: GPOWER: A general Power Analysis Program. Behavior Research Methods, Instruments, and Computers 28: 1-11.

Feuchter, Annette, 2001: Lob des sozialen Faulenzens. Motivation und Leistung beim Lösen komplexer Probleme in sozialen Situationen. Lengerich: Pabst Science Publishers.

Funke, Joachim, 2003: Problemlösendes Denken. Stuttgart: Kohlhammer.

William K. Gabrenya, Bibb Latané und Yue-Eng Wang, 1983: Social Loafing in Cross-cultural Perspective: Chinese on Taiwan. Journal of Cross-Cultural Psychology 14: 368-384.

Gabrenya, William K., Yue-Eng Wang und Bibb Latané, 1985: Social Loafing on an Optimizing Task: Cross-cultural Differences among Chinese and Americans. Journal of Cross-Cultural Psychology 16: 223-242.

Griffith, Terri L., Mark Fichman und Richard L.Moreland, 1989: Social Loafing and Social Facilitation: An Empirical Test of the Cognitive-motivational Model of Performance. Basic and Applied Social Psychology 10: 253-271.

Hackman, J. Richard, und Charles G. Morris, 1975: Groups that Work (and those that don't): Creating Conditions for Effective Teamwork. San Francisco, CA: Jossey-Bass.

Harkins, Stephen G., und Richard E. Petty, 1982: Effects of Task Difficulty and Task Uniqueness on Social Loafing. Journal of Personality and Social Psychology 43: 1214-1229.

Harkins, Stephen G., und Kate Szymanski, 1988: Social Loafing and Self-evaluation with an Objective Standard. Journal of Experimental Social Psychology 24: 354-365.

Harkins, Stephen G., und Kate Szymanski, 1989: Social Loafing and Group Evaluation. Journal of Personality and Social Psychology 56: 934-941.

Hertel, Guido, 2000: Motivation Gains in Groups: A Brief Review of the State of the Art. Zeitschrift für Sozialpsychologie 31: 169-175.

Hertel, Guido, Norbert L. Kerr und Lawrence A. Messé, 2000: Motivation Gains in Performance Groups. Paradigmatic and Theoretical Developments on the Köhler Effect. Journal of Personality and Social Psychology 79: 580-601.

Hill, Goldsmith W., 1982: Group versus Individual Performance: Are $\mathrm{n}+1$ Heads Better than One? Psychological Bulletin 91: 517-539.

Hoeksema-van Orden, Claudia Y. D., Anthony W. K. Gaillard und Bram P. Buunk, 1998: Social Loafing under Fatigue. Journal of Personality and Social Psychology 75: 1179-1190.

Jackson, J M., und Kipling D. Williams, 1985: Social Loafing on Difficult Tasks: Working Collectively can Improve Performance. Journal of Personality and Social Psychology 49: 937-942.

Karau, Steven J., und Kipling D. Williams, 1993: Social Loafing: A Meta-analytic Review and Theoretical Integration. Journal of Personality and Social Psychology 65: 681-706.

Karau, Steven J., und Kipling D. Williams, 1995: Social Loafing: Research Findings, Implications, and Future Directions. Current Directions in Psychological Science 4: 134-140.

Karau, Steven J., und Kipling D. Williams, 1997: The Effects of Group Cohesiveness on Social Loafing and Social Compensation. Group Dynamics 1: 156-168.

Karau, Steven J., und Kipling D. Williams, 2000: Understanding Individual Motivation in Groups: The Collective Effort model. S 113-141 in: Michael Edward Turner (Hg.), Groups at Work: Advances in Theory and Research. Mahwah, NJ: Erlbaum. 
Kravitz, David A., und Barbara Martin, 1986: Ringelmann Rediscovered: The Original Article. Journal of Personality and Social Psychology 50: 936-941.

Latané, Bibb, Kipling Williams und Stephen Harkins, 1979: Many Hands Make Light the Work: The Causes and Consequences of Social Loafing. Journal of Personality and Social Psychology 37: 822-832.

Nahavandi, Afsaneh, und Eileen Aranda, 1994: Restructuring Teams for the Re-engineered Organization. Academy of Management Executive 8: 58-68.

Olson, Mancur, 1992: Die Logik des kollektiven Handelns. Tübingen: Siebeck.

Omodei, Mary M., und Alex J. Wearing, 1993: Fire Chief User Manual. Melbourne: Department of Psychology: University of Melbourne.

Omodei, Mary M., und Alex J. Wearing, 1995a: Decision Making in Complex Dynamic Settings: A Theoretical Model Incorporating Motivation, Intention, Affect, and Cognitive Performance. Sprache und Kognition 14: 75-90.

Omodei, Mary M., und Alex J. Wearing, 1995b: The Fire Chief Microworld Generating Program: An Illustration of Computer-simulated Microworlds as an Experimental Paradigm for Studying Complex Decision-making Behavior. Behavior Research Methods, Instruments, und Computers 27: 303-316.

Ostrom, Elinor, 1998: A Behavioral Approach to the Rational Choice Theory of Collective Action. American Political Science Review 92: 1-22.

Parsons, Talcott, 1951: The Social System. New York: Free Press.

Reimer, Torsten, 2001: Kognitive Ansätze zur Vorhersage der Problemlöseleistung in Gruppen: Distraktion, Kompensation und Akzentuierung. Zeitschrift für Sozialpsychologie 32: 107-128.

Reimer, Torsten, Andrea Neuser und Clemens Schmitt, 1997: Unter welchen Bedingungen erhöht die Kommunikation zwischen den Gruppenmitgliedern die Koordinationsleistung in einer Kleingruppe? Zeitschrift für Experimentelle Psychologie 44: 495-518.

Schlenker, Barry R., und Mark R. Leary, 1982: Social Anxiety and Self-presentations: A Conceptualization and Model. Psychological Bulletin 14: 604-609.

Shepperd, James A., 1993: Productive Loss in Performance Groups: A Motivation Analysis. Psychological Bulletin 113: 67-81.

Shepperd, James A., und Kevin M. Taylor, 1999: Social Loafing and Expectancy-value Theory. Personality and Social Psychology Bulletin 25: 1147-1158.

Steiner, Ivan D., 1972: Group Process and Productivity. New York: Academic.

Szymanski, Kate, und Stephen G. Harkins, 1993: The Effect of Experimenter Evaluation on Selfevaluation within the Social Loafing Paradigm. Journal of Experimental Social Psychology 29: 268-286.

Vroom, Victor H., 1964: Work and Motivation. New York: Wiley und Sons.

Warnecke, Hans-Jürgen, 1997: Einleitung: Lernen durch Gruppen- und Teamarbeit. S. 1-3 in: Martin Kröll und Herbert Schnauber (Hg.), Lernen der Organisation durch Gruppen- und Teamarbeit. Berlin: Springer.

Wicklund, Richard A. (1975). Objective Self-awareness. S. 233-275 in: Leonard Berkowitz (Hg.), Advances in Experimental Social Psychology. New York: Academic Press.

Williams, Kipling D., Steve A. Nida, Lawrence D. Baca und Bibb Latané, 1989: Social Loafing and Swimming: Effects of Identifiability on Individual and Relay Performance of Intercollegiate Swimmers. Basic and Applied Social Psychology 10: 73-81.

Yerkes, Robert M., und J. D. Dodson, 1908: The Relation of Strength of Stimulus to Rapidity of Habit Formation. Journal of Comparative and Neurological Psychology 18: 459-482.

Korrespondenzadresse: Prof. Dr. Joachim Funke, Psychologisches Institut der Universität Heidelberg, Hauptstr. 47-51, D-69117 Heidelberg

E-Mail: joachim.funke@psychologie.uni-heidelberg.de 